\title{
Chloride Penetration in Circular Concrete Columns
}

\author{
M. Morga ${ }^{1)}$, and G. C. Marano ${ }^{2), *}$
}

(Received October 10, 2013, Accepted December 8, 2014, Published online February 4, 2015)

\begin{abstract}
Most of the diffusion models of chloride ions in reinforced concrete (RC) elements proposed in literature are related to an isotropic homogeneous semi-infinite medium. This assumption reduces the mathematical complexity, but it is correct only for plane RC elements. This work proposes a comparison between the diffusion model of chloride ions in RC circular columns and in $\mathrm{RC}$ slab elements. The durability of RC cylindric elements estimated with the circular model instead of the plane model is shown to be shorter. Finally, a guideline is formulated to properly use the standard and more simple plane model instead of the circular one to estimate the time to corrosion initiation of cylindrical RC elements.
\end{abstract}

Keywords: chloride ions diffusion, circular column, time to corrosion initiation, pitting corrosion of rebars.

\section{List of Symbols \\ $\operatorname{erf}(\bullet)$ \\ $t$ \\ $t_{c r}$ \\ $x$ \\ $w / c$ \\ $C(x, t)$ or $C\left(\rho_{1}, t\right)$}

$C_{0}$

$C_{c r}$

$D\left(\mathrm{~cm}^{2} /\right.$ year $)$

$R$

$\rho$

$\rho_{1}=R-\rho$

$\operatorname{erf}\left(\frac{x}{2 \sqrt{D t}}\right)$
Error function

Time from the beginning of the exposure to chloride solution

Time to corrosion initiation

Perpendicular distance from external surface in the slab model

Water-cement ratio

Chloride concentration in $\mathrm{RC}$ structural member at the distance $x$ or $\rho_{1}$ from the external surface at time $t$ from the first exposure to chloride solution

Chloride concentration on the external surface of the RC structural member

Chloride threshold concentration for the beginning of the depassivation

Diffusion coefficient

Radius of the circular cross-section Radial distance from the centre in a circular model

Radial distance from the external surface in a circular model

Diffusion element of the slab model

\footnotetext{
${ }^{1)}$ Mobility Department - Transportation Infrastructures Technologies, AIT Austrian Institute of Technology, Vienna, Austria.

${ }^{2)}$ Department of Civil Engineering and Architecture, Technical University of Bari, Bari, Italy.

*Corresponding Author; E-mail: g.c.marano@gmail.com Copyright $($ The Author(s) 2015. This article is published with open access at Springerlink.com
}

$\begin{array}{ll}\frac{2}{R} \sum_{m=1}^{\infty} \frac{1}{\alpha_{m}} \frac{J_{0}\left(\rho \alpha_{m}\right)}{J_{1}\left(R \alpha_{m}\right)} e^{\left(-D \alpha_{m}^{2} t\right)} & \begin{array}{l}\text { Diffusion element of the circular } \\ \text { model }\end{array} \\ J_{0}\left(\rho \alpha_{m}\right) & \begin{array}{l}\text { Bessel's first function with order } \\ \text { zero } \\ \text { Bessel's first function with order } \\ J_{1}\left(R \alpha_{m}\right)\end{array} \\ \text { one }\end{array}$

\section{Introduction}

The reinforced concrete (RC) is one of the most used construction materials. During the hydration process the $\mathrm{pH}$ becomes highly alkaline inside the concrete matrix due to the formation of the products of the cement hydration. This alkaline $\mathrm{pH}$ produces a passive film of iron oxide or hydroxide on the surface of the steel bars that protect the bars themselves from the corrosion. Therefore, in RC element the reinforcements are not corroded until this layer stays. During its service life a RC structure may be exposed to aggressive species that migrate inside the concrete from their exposed surface and reduce the alkaline protective $\mathrm{pH}$. When the concentration of these aggressive species reaches a threshold value and consequently the $\mathrm{pH}$ in the matrix concrete is sufficiently low, the oxidation of the steel bars begins. This process is referred to as "depassivation" (Val and Trapper 2008; Martin-Perez et al. 2001; Saetta et al. 1995; Izquierdo et al. 2004) of the reinforcement bars. The reduction of the $\mathrm{pH}$ in the concrete matrix is produced by two main mechanisms: the $\mathrm{CO}_{2}$ diffusion from the atmosphere and the diffusion of active ions, like chloride ions (Martin-Perez et al. 2001; Saetta et al. 1995). The diffusion of chloride ions is the principal cause of the reinforcement bar corrosion in marine environment or cold climates.

Chloride ions are sometimes present inside the hydration water used to mix the concrete. In that case these ions slow down or prevent the formation of the protective passive layer 
on the steel surface. In the other cases the chloride ions migrate into the contrete matrix from the external enviroment. The time at which the critical chloride concentration is reached on the passive layer is called "time to initiation", according to the Tuutti model (Tuutti 1982) (see Fig. 1). This time devides the initiation phase from the propagation phase. During the initiation phase the chloride ions diffuse in the concrete matrix, but the steel corrosion is absent. In the propagation phase the corrosion started and proceed to a critical value of steel loss that causes failure of the structural element or all the structure. The propagation phase is considerably shorter than the initiation phase. Therefore, the prediction of the time to corrosion initiation is an important aspect in reliability analysis of RC structures because it indicates the beginning of the strenght reduction of the structural elements (Nogueira and Leonel 2013; Marano et al. 2008). For this reason the estimation of the time to corrosion initiation has been investigated by several researchers.

The chloride penetration in the concrete matrix occurs through either permeation and absorbition of a chloride solution or diffusion of free chloride ions into the saturated concrete (Val and Trapper 2008; Martin-Perez et al. 2001). These mechanisms may also occur sequentially. The absorption runs out in short time and after that the diffusion begins due to the saturation of the concrete pores (MartinPerez et al. 2001). Each transport process is modelled by a different mathematical law depending on the forces that are at stake. In the convection process the motion of the solution inside the concrete pores (and then the motion of the chloride ions) is caused by the moisture/humidity gradient. The gradient of chloride concentration between the external surface and the inside of saturated concrete matrix causes the chloride ions motion in the diffusion process (Val and Trapper 2008; Nogueira and Leonel 2013; Basheer et al. 2002). This paper proposes the investigation of the chloride ingress in piers partially submerged in seawater, but fully saturated: the part of the pier out of the water is subject to continuous wetting cycles, therefore it is constantly wet and saturated. In these conditions the diffusion phenomenon is prevalent. The diffusion process of chloride ions in the concrete matrix is not irreversible in case of inversion of the concentration gradient and is history independent, so it is a normal diffusion process (Nogueira and Leonel 2013). For that reason the Fick's Second Law is used to model the timevariant chloride ions diffusion. The validity of the Fick's second law to model the diffusion of chloride ions in concrete is determined on an empirical basis (Chatterji 1995).

Different elements influence the chloride penetration into $\mathrm{RC}$ elements as combination of air or water pressure, humidity and concetration differences or temperature differences of solutions (Izquierdo et al. 2004; Nogueira and Leonel 2013; Basheer et al. 2002). Some studies investigated the influence of the different factors on the chloride threshold value that lead to the depassivation of the rebars (Izquierdo et al. 2004; Azad 1998). Most of those studies are based on the simple plane monodirectional diffusion model, called "slab model". In this model the concrete matrix is assumed to be an isotropic homogeneous semi-infinite medium with higher chloride concentration on its external plane surface than the inside concentration. In this model the concentration gradient causes the diffusion of chloride ions inside the concrete matrix along the direction perpendicular to the external surface. The Fick's Second Law (Chatterji 1995) describing the diffusion process in this slab model depend only on one spatial variable $x$ and the time $t$ (Collepardi et al. 1972):

$$
\left(\frac{\partial C}{\partial t}\right)=D \frac{\partial^{2} C}{\partial x^{2}}
$$

The spatial variable $x$ is the distance of the inside point, where the concentration is estimated, from the external surface, while the constant $D$ is the diffusion coefficient. During the diffusion process the chloride concentration $C$ changes inside the concrete matrix and the concentration gradient decreases.

The parameters affecting the chloride diffusion inside the concrete matrix are initial chloride concentration in the concrete mix, type of cement used in the mix, water-cement ratio ( $w / c$ ratio), curing conditions, external temperature and external concentration of the chloride ions and variation of the external concentration in time. The effect of the type of cement, the $w / c$ ratio and the curing conditions on the chloride diffusion are all included in the value of the diffusion coefficient $D\left(\right.$ in $\left.\mathrm{m}^{2} / \mathrm{s}\right)$ (Azad 1998). In the most simple studies the chloride diffusion coefficient is kept constant

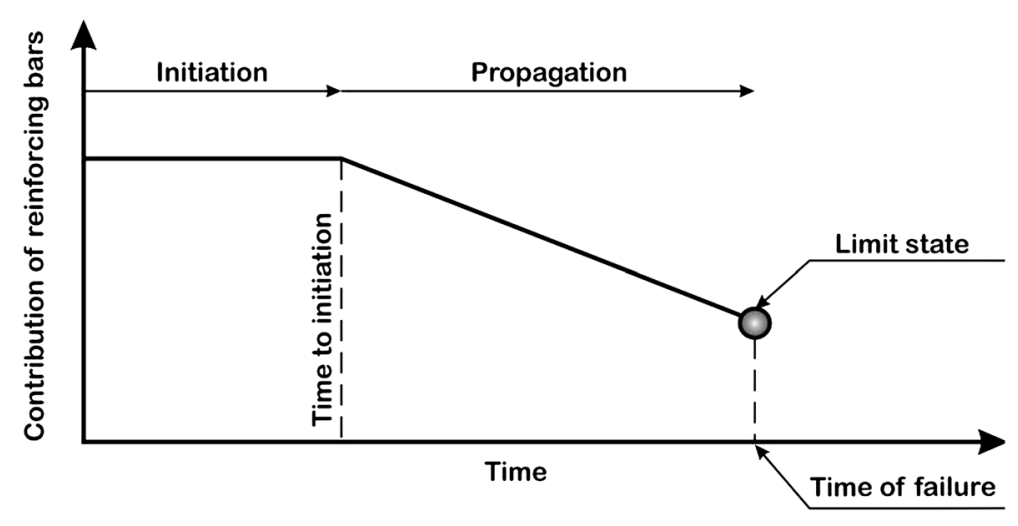

Fig. 1 Deterioration in reinforcing bar due to corrosion process. 


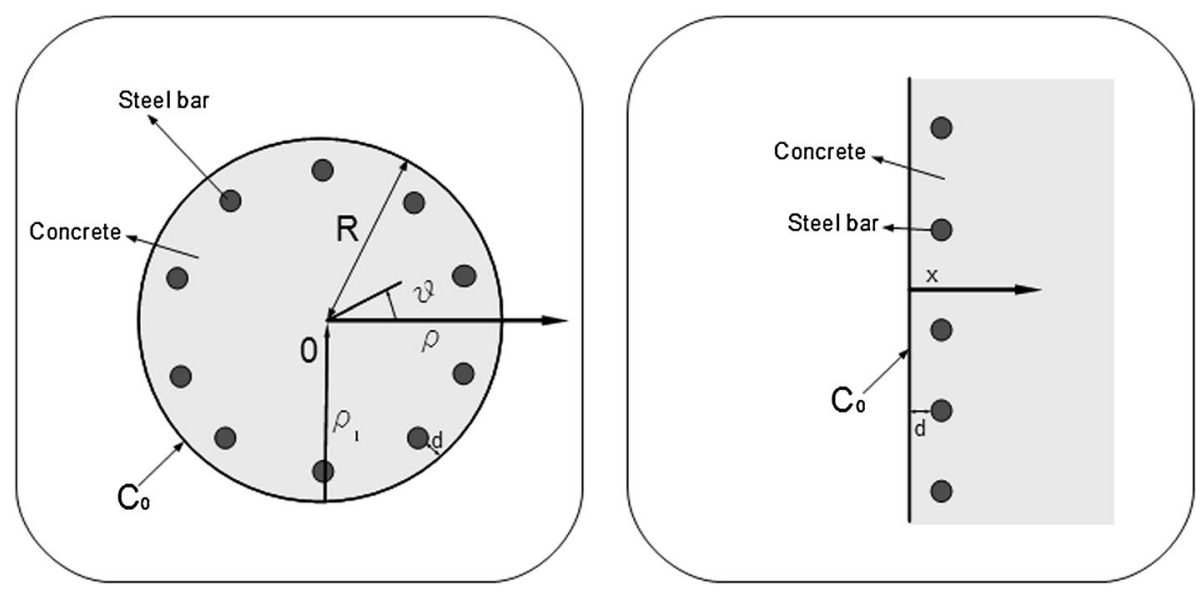

Fig. 2 Left cross-section of a circular RC column; right cross-section of an RC semi-infinite plane member.

during the diffusion process (Collepardi et al. 1972). Some scholars presented works in which the diffusion coefficient is estimated as a function of different parameters, like the $w / c$ ratio (Lin 1990). Different laws to estimate the diffusion coefficient of ordinary portland concrete (OPC) Type I were compared in ( $\mathrm{Vu}$ and Stewart 2000). The diffusion coefficients estimated according to these laws resulted to be similar for $w / c$ ratio in the range $0.3-0.5$. Furthermore the diffusion coefficient is highly dependent from the temperature, but the $w / c$ ratio influences the value of this coefficient in the same way at different temperatures, as showed in (Kirkpatrick et al. 2002). Other studies demonstrated the dependence of diffusion coefficient on the age the temperature and the humidity at the same time (Val and Trapper 2008; Martin-Perez et al. 2001). It is important to notice that in literature the diffusion coefficient is always evaluated by assuming the occurrence of diffusion in a semi-infinite medium with plane external surface. This assumption is questionable in case the estimation of the diffusion coefficient is supported by experimental data: both laboratory samples and several real RC structural member from which samples are taken are not semi-infinite plane elements.

Although the deterministic approach to investigate the chloride diffusion in RC structural members is the most adopted, some studies proposed a probabilistic assessment of the time to corrosion initiation in $\mathrm{RC}$ elements. The timedependent probability of steel corrosion initiation in partially saturated RC members after exposure to chloride ions is calculated in (Val and Trapper 2008) by taking into account both the diffusion and convection phenomena. In (Nogueira and Leonel 2013) and (Saassouh and Lounis 2012) a probabilistic model of chlorides diffusion is coupled to reliability algorithms to determine the probability of failure of an RC structure. Both numerical approach, like Monte Carlo simulation, (Nogueira and Leonel 2013; Saassouh and Lounis 2012) and analytical approaches, like FORM (Nogueira and Leonel 2013; Saassouh and Lounis 2012) and SORM (Saassouh and Lounis 2012), were tested by scholars to estimate the limit state of corrosion in RC structures. The uncertainties affecting the diffusion coefficient $\mathrm{D}$ have a large scatter in the first life years of an RC structure, while after that time the overall uncertainty of the diffusion coefficient results reduced (Saassouh and Lounis 2012). The time and space invariant diffusion coefficient is an questionable assumption, but it is often supported by measures on the real concrete elements (Saassouh and Lounis 2012). It is clear that some uncertainty could be reduced by increasing the knowledge of the corrosion mechanism or testing the structure: in this case the uncertainty should be modelled as epistemic instead of aleatory (Saassouh and Lounis 2012; Do et al. 2005). The different kind of uncertainty could be handled by random, fuzzy or fuzzy random variables (Sobhani and Ramezanianpour 2011). In (Do et al. 2005) the uncertainty of the parameters on which the time to corrosion initiation depends is treated with the fuzzy logic. The defuzzification of the time to corrosion initiation could be considered a deterministic value to use in the maintenance planning of the structure. The fuzzy logic is an effective tool also in case the model defined to assess the reliability of RC structures subject to pitting reinforcement corrosion depends on both probabilistic and nonprobabilistic parameters (Marano et al. 2008). In complex models for the life cycle prediction and service life design of RC structures the uncertainties of the model parameters and the degradation mechanisms are not negligible. The interdependence among both parameters and mechanisms is also critical for the life cycle prediction of RC structure, therefore also in the assessment of the time to corrosion initiation. In (Sobhani and Ramezanianpour 2011) the high complexity of this problem depending on several parameters modelled as fuzzy random variables and interdependent mechanisms was managed by proposing an algorithm with a soft computing core.

In previous deterministic studies (Val and Trapper 2008; Tuutti 1982; Basheer et al. 2002) the time to corrosion initiation is evaluated with the Fick's second law formulated for the slab model (Eq. (1)). The solution of this equation is (Crank 1975)

$$
C(x, t)=C_{0}\left[1-\operatorname{erf}\left(\frac{x}{2 \sqrt{D t}}\right)\right]
$$


where $\operatorname{erf}(\bullet)$ is the error function, $C_{0}$ is the chloride concentration on the external surface (expressed in $\mathrm{kg} / \mathrm{m}^{3}$ or in $\%), D$ is the apparent chloride diffusion coefficient and $t$ is the duration of the exposure to chlorides (Tang and Joost 2007). Assuming D as time-invariant, the complement of the error function $(1-\operatorname{erf}(\bullet))$ describes the evolution of the chloride diffusion front in a monodimensional model in dependence to the exposure time $t$ (Tang and Joost 2007; Tuutti 1982) and the distance from the external surface $x$.

Generally, the solution of the Fick's second law depends on the geometry of the element where the diffusion occurs, as well on the shape of the source (Crank 1975). Therefore, the time to corrosion initiation due to chloride ions diffusion depends on the geometry of the RC element. Some scholars proposed solutions of the Fick's second law for aggressive species in the two-dimensional (2-D) models (Val and Trapper 2008; Martin-Perez et al. 2001; Frier and Sørensen 2007) and one-dimensional not plane (1-D) model of RC elements (Arora et al. 1997). First Martin-Perez et al. (2001) and after Val et al. (Val and Trapper 2008) used a 2-D ingress of chloride ions into partially saturated RC members with rectangular cross-section. In these studies the chloride penetration is due to both the diffusion and the convection, while in (Frier and Sørensen 2007) only the chloride diffusion is estimated. In these 2-D chloride diffusion models it is assumed that the chloride ions penetrate at the same into the concrete section time along two directions perpendicular to the exposed external surfaces. This kind of model is applicable to RC elements with rectangular cross-section. Val and Trapper (2008) proved that the total chloride content increases faster near the reinforcement in a corner of a 2-D cross-section than in the proximity of the rebar in a 1-D model. For this reason the life-time of a rectangural RC element is shorter than the life-time of a $\mathrm{RC}$ element with geometry approximable to a plane semi-infinite element.

The piers cross-section of bridges or quays could be not only rectangular, but also circular. In (Arora et al. 1997) the chloride diffusion process was investigated for columns with a circular cross-section. In that article the diffusion in a model of one steel bar covered by saturated concrete and exposed to a chloride solution was analysed. Sensitivity analyses for different initial and boundary conditions were made. Finally, the analytical results were compared with experimental data taken on circular columns with radii smaller than ones used in real structures. Although that article proposed the solution of the Fick's second law for element with circular cross section, the results were estimated for $\mathrm{RC}$ members with unrealistic dimensions.

This paper proposes the analytical solution of Fick's second law for RC members with circular cross section calculated for RC cylindrical columns with realistic dimensions. These results are compared with the results of the chloride diffusion in the slab model to highlight the difference between the time to corrosion initiation of the RC elements estimated with the model of a cylindrical column and the time estimated with the semi-infinite plane model. This comparison leads to indications about the limit of use the simple "slab model" to estimate the time to corrosion initiation in case of $\mathrm{RC}$ members with circular cross-section.

\section{Chloride Diffusion in a Cylindrical Column}

For the 1-D semi-infinite chloride diffusion model the Fick's Second Law is function of only one spatial variable $x$ expressed in Cartesian coordinates. This variable corresponds to the direction perpendicular to the external surface exposed to the chloride solution (Crank 1975). In 2-D models of the chloride diffusion into $\mathrm{RC}$ elements the ions propagate along two different directions perpendicular to the external surfaces exposed to the chloride solution. In this case the Fick's Second Law is function of two spatial variables expressed in Cartesian coordinates (Crank 1975) ( $x$ and $y)$ :

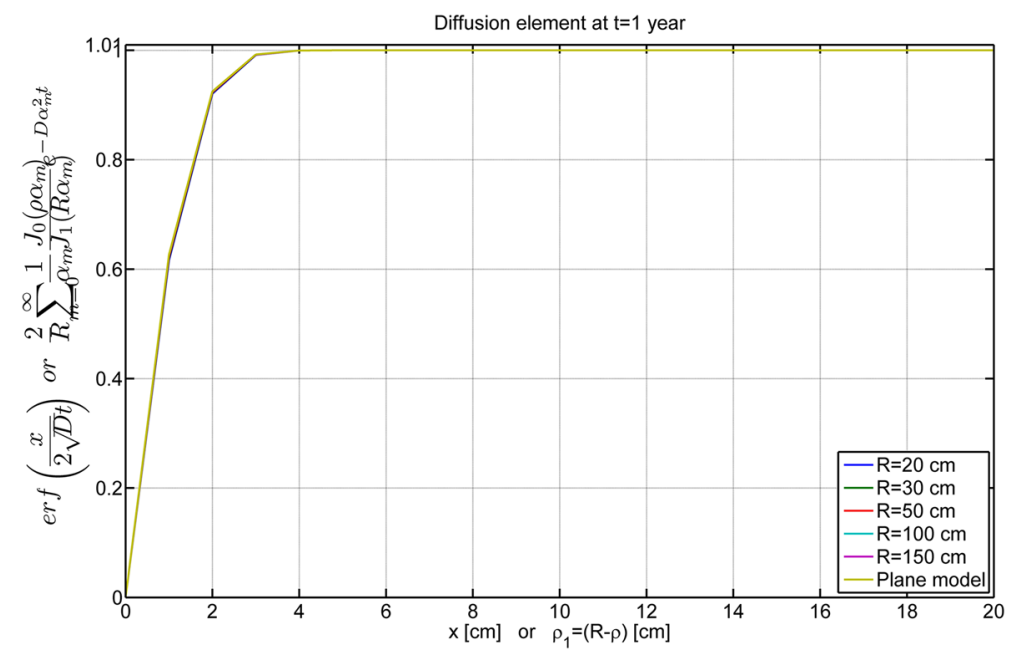

Fig. 3 "Diffusion elements" of the slab model and the circular model. The values of the diffusion element of the circular model is estimated for columns characterized by radii in the range $(20-150 \mathrm{~cm})$. The diffusion elements are evaluated at time 1 year from the first exposure of the concrete matrix to chloride ions and for different distance $\left(x\right.$ and $\left.\rho_{1}\right)$ in the concrete matrix from the exposed external surface. 


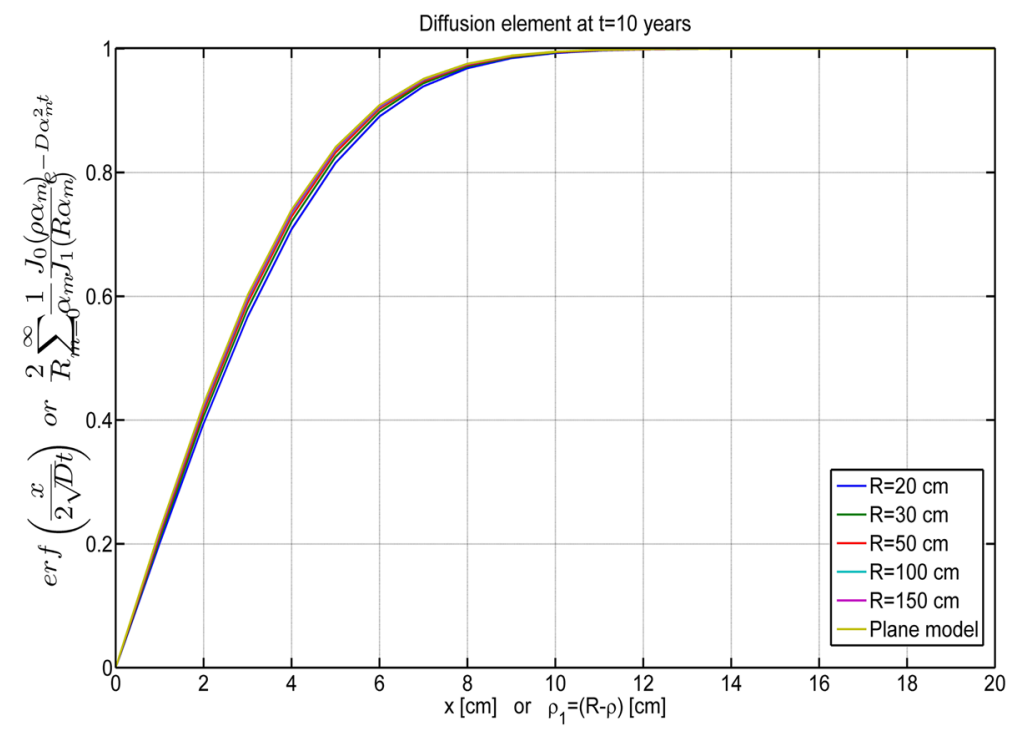

Fig. 4 "Diffusion elements" of the slab model and the circular model. The values of the diffusion element of the circular model is estimated for columns characterized by radii in the range $(20-150 \mathrm{~cm})$. The diffusion elements are evaluated at time 10 year from the first exposure of the concrete matrix to chloride ions and for different distance $\left(x\right.$ and $\left.\rho_{1}\right)$ in the concrete matrix from the exposed external surface.

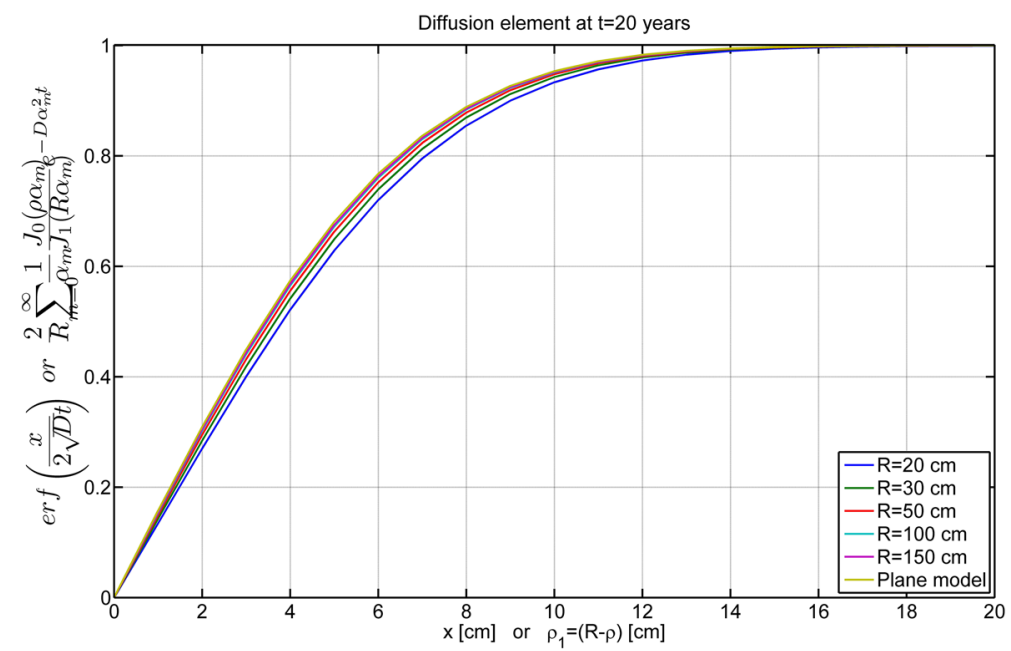

Fig. 5 "Diffusion elements" of the slab model and the circular model. The values of the diffusion element of the circular model is estimated for columns characterized by radii in the range $(20-150 \mathrm{~cm})$. The diffusion elements are evaluated at time 20 year from the first exposure of the concrete matrix to chloride ions and for different distance $\left(x\right.$ and $\left.\rho_{1}\right)$ in the concrete matrix from the exposed external surface.

$$
\left(\frac{\partial C}{\partial t}\right)=D\left(\frac{\partial^{2} C}{\partial x^{2}}+\frac{\partial^{2} C}{\partial y^{2}}\right)
$$

The chloride diffusion in RC elements with circular crosssection is described by the Fick's Second Law expressed in cylindrical coordinates

$$
\left(\frac{\partial C}{\partial t}\right)=D\left(\frac{1}{\rho} \frac{\partial}{\partial \rho}\left(\rho \frac{\partial C}{\partial \rho}\right)+\frac{1}{\rho^{2}} \frac{\partial^{2} C}{\partial \theta^{2}}+\frac{\partial^{2} C}{\partial z^{2}}\right)
$$

The RC cylindrical elements are axial-symmetrical, so in them the chloride diffusion occurs along the radial direction from the external surface exposed to the chloride concentration $C_{0}$ to the inside of the concrete matrix.
Therefore, the Fick's Second Law expressed with cylindrical coordinates (4) becomes (Crank 1975)

$$
\frac{\partial C}{\partial t}=D\left(\frac{1}{\rho} \frac{\partial C}{\partial \rho}+\frac{\partial^{2} C}{\partial \rho^{2}}\right)
$$

where the chloride concentration depends only on the time and one spatial variable: the radial coordinate $\rho$ (Fig. 2).

The boundary conditions in the cylindrical model are

$$
\begin{array}{ll}
C(R, t)=C_{0} & t \in[0,+\infty] \\
\frac{\partial(C(0, t))}{\partial \rho}=0 & t \in[0,+\infty]
\end{array}
$$




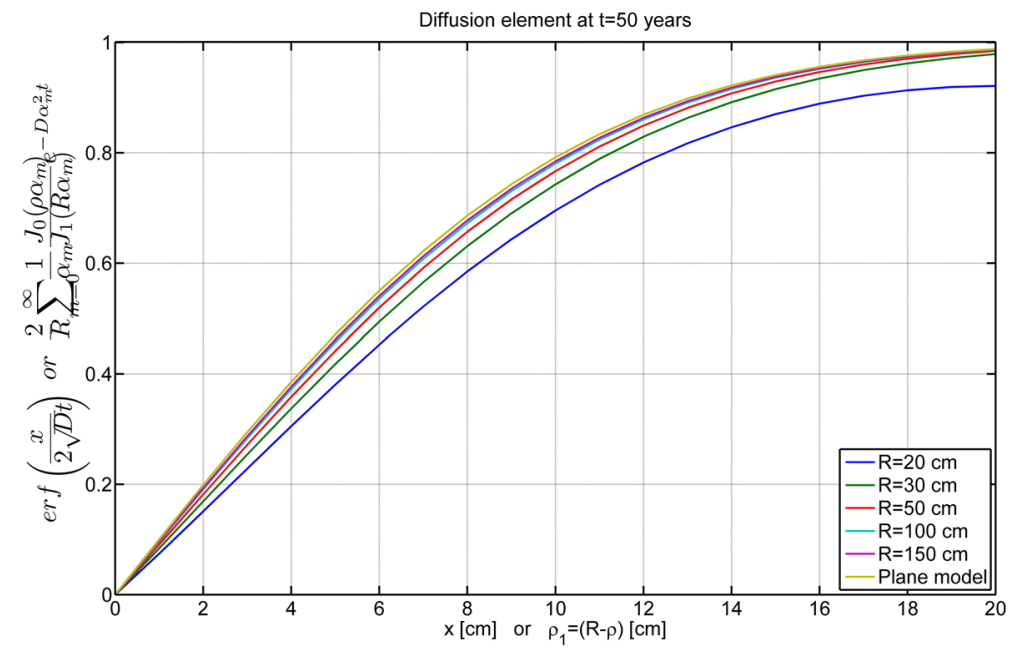

Fig. 6 Diffusion elements of the slab model and the circular model. The values of the diffusion element of the circular model is estimated for columns characterized by radii in the range $(20-150 \mathrm{~cm})$. The diffusion elements are evaluated at time 50 year from the first exposure of the concrete matrix to chloride ions and for different distance $\left(x\right.$ and $\left.\rho_{1}\right)$ in the concrete matrix from the exposed external surface.
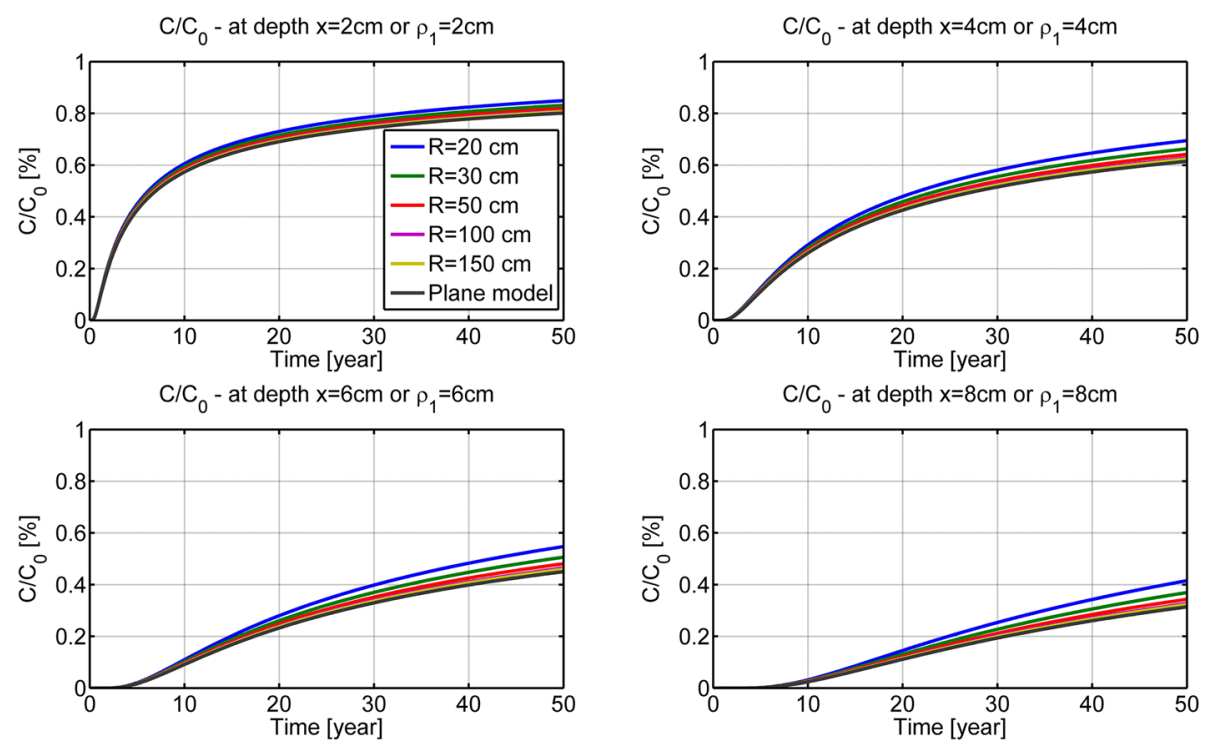

Fig. 7 Ratio of the chloride concentration at depth $x$ or $\rho_{1}$ from the external exposed surface $C$ and the chloride concentration on the external surface $C_{0}$ as function of time of exposure to chloride $t$. Value extimated for slab model and circula model characterized by radii in the range $(20-150 \mathrm{~cm})$.

Table 1 Ratio of the chloride concentration in a circular RC cross-section and the chloride concentration of the plane RC element $C / C_{\text {slab }}$ estimated at depth $\rho_{1}=2 \mathrm{~cm}$ from the external surface.

\begin{tabular}{c|c|c|c|c|c}
\hline$C / C_{\text {slab }}$ & $t=1$ year & $t=5$ years & $t=10$ years & $t=20$ years & $t=50$ years \\
\hline \hline$R=20 \mathrm{~cm}$ & 1,054 & 1,055 & 1,056 & 1,057 & 1,060 \\
\hline$R=30 \mathrm{~cm}$ & 1,035 & 1,036 & 1,036 & 1,036 & 1,037 \\
\hline$R=50 \mathrm{~cm}$ & 1,021 & 1,021 & 1,021 & 1,021 & 1,021 \\
\hline$R=100 \mathrm{~cm}$ & 1,010 & 1,010 & 1,010 & 1,010 & 1,010 \\
\hline$R=150 \mathrm{~cm}$ & 1,007 & 1,007 & 1,007 & 1,007 & 1, \\
\hline
\end{tabular}


Table 2 Ratio of the chloride concentration in a circular RC cross-section and the chloride concentration of the plane RC element $C / C_{\text {slab }}$ estimated at depth $\rho_{1}=4 \mathrm{~cm}$ from the external surface.

\begin{tabular}{c|c|c|c|c|c}
\hline$C / C_{\text {slab }}$ & $t=1$ year & $t=5$ years & $t=10$ years & $t=20$ years & $t=50$ years \\
\hline \hline$R=20 \mathrm{~cm}$ & 1,119 & 1,120 & 1,121 & 1,124 & 1,130 \\
\hline$R=30 \mathrm{~cm}$ & 1,074 & 1,075 & 1,075 & 1,076 & 1,078 \\
\hline$R=50 \mathrm{~cm}$ & 1,043 & 1,043 & 1,043 & 1,043 & 1,044 \\
\hline$R=100 \mathrm{~cm}$ & 1,021 & 1,021 & 1,021 & 1,021 & 1,021 \\
\hline$R=150 \mathrm{~cm}$ & 1,014 & 1,014 & 1,014 & 1,014 & 1,014 \\
\hline
\end{tabular}

Table 3 Ratio of the chloride concentration in a circular RC cross-section and the chloride concentration of the plane RC element $C / C_{\text {slab }}$ estimated at depth $\rho_{1}=6 \mathrm{~cm}$ from the external surface.

\begin{tabular}{c|c|c|c|c|c}
\hline$C / C_{\text {slab }}$ & $t=1$ year & $t=5$ years & $t=10$ years & $t=20$ years & $t=50$ years \\
\hline \hline$R=20 \mathrm{~cm}$ & 1,196 & 1,198 & 1,200 & 1,204 & 1,216 \\
\hline$R=30 \mathrm{~cm}$ & 1,118 & 1,119 & 1,120 & 1,121 & 1,124 \\
\hline$R=50 \mathrm{~cm}$ & 1,066 & 1,066 & 1,067 & 1,067 & 1,068 \\
\hline$R=100 \mathrm{~cm}$ & 1,031 & 1,031 & 1,032 & 1,032 & 1,032 \\
\hline$R=150 \mathrm{~cm}$ & 1,021 & 1,021 & 1,021 & 1,021 & 1,021 \\
\hline
\end{tabular}

Table 4 Ratio of the chloride concentration in a circular RC cross-section and the chloride concentration of the plane RC element $C / C_{\text {slab }}$ estimated at depth $\rho_{1}=8 \mathrm{~cm}$ from the external surface.

\begin{tabular}{c|c|c|c|c|c}
\hline$C / C_{\text {slab }}$ & $t=1$ year & $t=5$ years & $t=10$ years & $t=20$ years & $t=50$ years \\
\hline \hline$R=20 \mathrm{~cm}$ & - & 1,295 & 1,298 & 1,304 & 1,323 \\
\hline$R=30 \mathrm{~cm}$ & - & 1,169 & 1,170 & 1,172 & 1,176 \\
\hline$R=50 \mathrm{~cm}$ & - & 1,091 & 1,092 & 1,092 & 1,093 \\
\hline$R=100 \mathrm{~cm}$ & - & 1,043 & 1,043 & 1,043 & 1,043 \\
\hline$R=150 \mathrm{~cm}$ & - & 1,028 & 1,028 & 1,028 & 1,028 \\
\hline
\end{tabular}

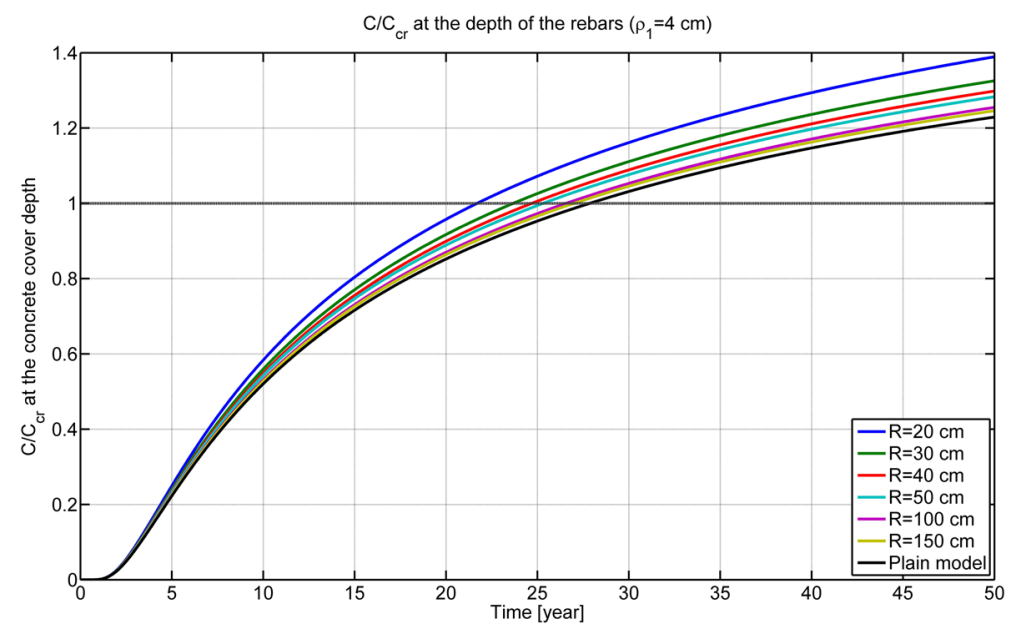

Fig. 8 Ratio of the chloride concentration estimated at the depth of rebars in the concrete matrix $\left(x\right.$ or $\left.\rho_{1}=4 \mathrm{~cm}\right) C$ and the chloride threshold concentration for the beginning of the depassivation $C_{C r}$ as function of the exposure time $t$. The horizontal black line indicate the reaching of the threshold chloride, so the beginning of the depassivation of the rebars. (Color figure online). 
Table 5 Time to corrosion initiation of circular cross-section or plane element.

\begin{tabular}{c|c|c|c|c|c|c|c}
\hline Time [year] & $R=20 \mathrm{~cm}$ & $R=30 \mathrm{~cm}$ & $R=40 \mathrm{~cm}$ & $R=50 \mathrm{~cm}$ & $R=100 \mathrm{~cm}$ & $R=150 \mathrm{~cm}$ & Slab \\
\hline \hline$w / c=0.50$ & 21.8 & 23.7 & 24.7 & 25.4 & 26.6 & 27.1 & 27.9 \\
\hline$w / c=0.45$ & 43.5 & 47.4 & 49.4 & 50.7 & 53.2 & 54.1 & 55.8 \\
\hline$w / c=0.40$ & 86.9 & 94.8 & 98.8 & 101.3 & 106.4 & 108.1 & 111.5 \\
\hline
\end{tabular}
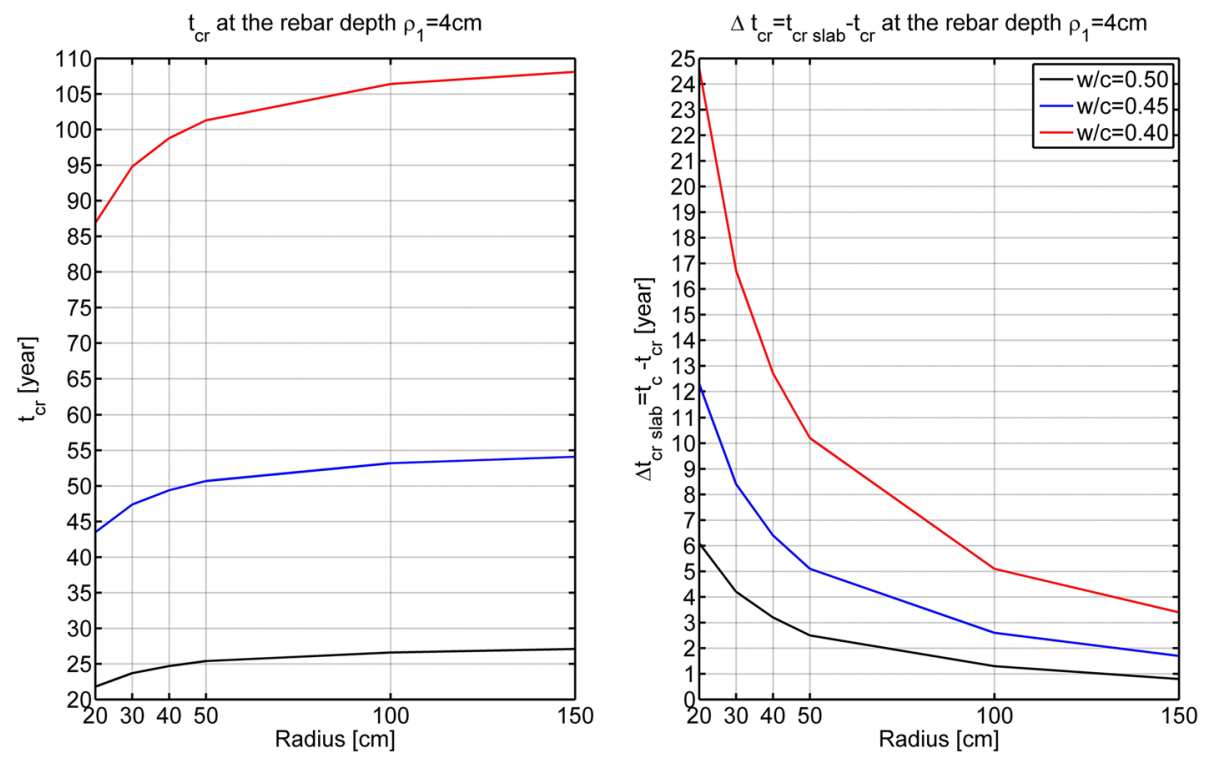

Fig. 9 Left time to corrosion initiation $t_{C r}$ of the rebars rebar estimated for circular model as function of the radius of cross-section $R$. Calculation proposed for different water-concrete ratios $w / c$. Right difference between the time to corrosion initiation of a cylindrical RC column and the time to corrosion initiation of the plane RC element $\Delta t_{C r-s l a b}$ as function of the radius of the circular cross-section $R$.

Table 6 Difference of the critical time to corrosion initiation $\Delta t_{C r-s l a b}$ of the RC plane element and of the RC element with circular cross-section $t_{\text {Cr-circular }}$.

\begin{tabular}{c|c|c|c|c|c|c}
\hline$\Delta t$ (year) & $R=20 \mathrm{~cm}$ & $R=30 \mathrm{~cm}$ & $R=40 \mathrm{~cm}$ & $R=50 \mathrm{~cm}$ & $R=100 \mathrm{~cm}$ & $R=150 \mathrm{~cm}$ \\
\hline \hline$w / c=0.50$ & 6.1 & 4.2 & 3.2 & 2.5 & 1.3 & 0.8 \\
\hline$w / c=0.45$ & 12.3 & 8.4 & 6.4 & 5.1 & 2.6 & 1.7 \\
\hline$w / c=0.40$ & 24.6 & 16.7 & 12.7 & 10.2 & 5.1 & 3.4 \\
\hline
\end{tabular}

while the initial condition is

$$
C(\rho, 0)=f(\rho) \quad \rho \in] 0, R[
$$

The first boundary condition (Eq. (6)) defines a constant chloride concentration $C_{0}$ on the external surface (where $\rho=R$ ), while the second one (Eq. (7)) is related to the axialsymmetry of the diffusion. The initial condition (Eq. (8)) defines the chloride concentration inside the RC column at the time of the first concrete exposure to the chloride solution.

Equation (5) with the boundary conditions (Eqs. (6) and (7)) and the initial condition (Eq. (8)) can be solved as indicated in the appendix. The solution is

$$
C=C_{0}\left(1-\frac{2}{R} \sum_{m=1}^{\infty} \frac{1}{\alpha_{m}} \frac{J_{0}\left(\rho \alpha_{m}\right)}{J_{1}\left(R \alpha_{m}\right)} e^{\left(-D \alpha_{m}^{2} t\right)}\right)
$$

where $J_{n}$ is Bessel's first function and $\alpha_{m}$ depends on it.

\section{Comparison Between Cylindrical Model and Slab Model}

As afore-said, the depassivation process of the rebars occurs when the chloride concentration in the concrete matrix at the depth of the rebar location exceeds the threshold concentration $C_{\mathrm{cr}}$ :

- For the cylindrical columns $C\left(t_{c r}, \rho_{s}\right)=C_{c r}$

- For the element with plane surface $C\left(t_{c r}, x_{s}\right)=C_{c r}$

Clearly, the circular model is equivalent to the slab model for $R \rightarrow \infty$.

In the slab model the chloride concentration at depth $x$ and time $t$ depends on the error function:

$$
\operatorname{erf}\left(\frac{x}{2 \sqrt{D t}}\right)
$$




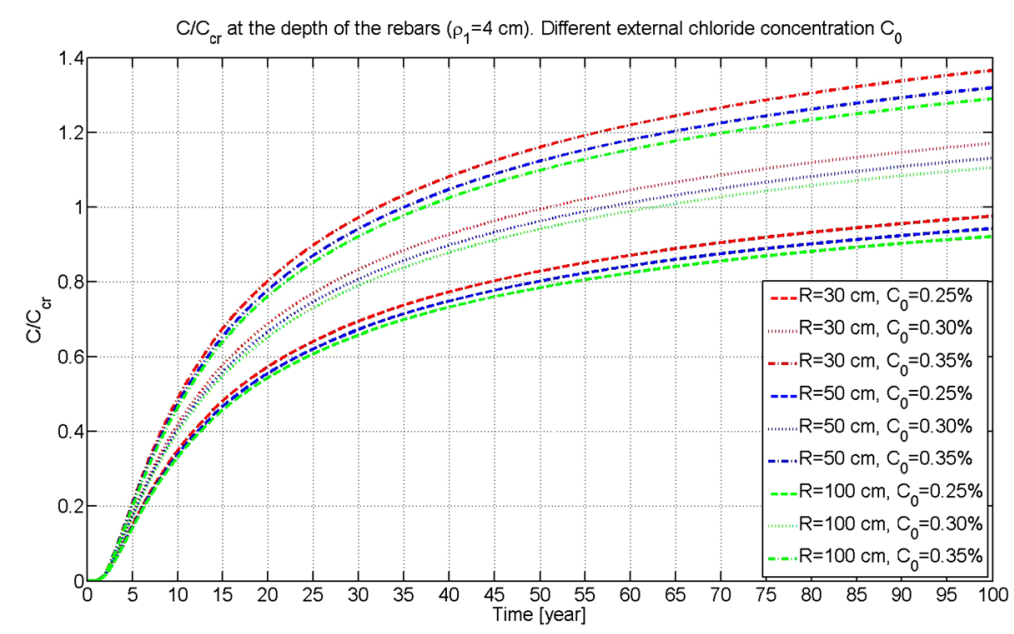

Fig. 10 The ratio of chloride concentration at the depth of steel bars in the concrete matrix $C$ and the threshold chloride concentration $C_{c r}$ as function of the exposure time $t$. Calculation made for chloride concentrations on the external surface $C_{0}=[0.25,0.30,0.35 \%]$ and radii of the circular cross section $R=[30,50,100 \mathrm{~cm}]$.

In the circular model the chloride concentration at depth $\rho$ at the time $t$ is a function of the sum of products of an exponential term and a ratio of Bessel's first functions:

$$
\frac{2}{R} \sum_{m=1}^{\infty} \frac{1}{\alpha_{m}} \frac{J_{0}\left(\rho \alpha_{m}\right)}{J_{1}\left(R \alpha_{m}\right)} e^{\left(-D \alpha_{m}^{2} t\right)}
$$

To be compared to Eq. (2), Eq. (9) should be expressed in term of $\rho_{1}=R-\rho$, i.e. depth from the external surface, instead of $\rho$, i.e. distance from the centre of the cross section. Hereafter both Eqs. (10) and (11) are called "diffusion element". In the first part of the study the diffusion coefficient used is $D=0.63 \mathrm{~cm}^{2} /$ year: it is a characteristic value for the young OPC Type I with cement-ratio $w /$ $c=0.5$ and compressive strength of 30-40 MPa.

Figures 3, 4, 5 and 6 show the values of the diffusion element estimated for both the slab model and the circular model with different radii as function of depth in the concrete element from the external surface. In these figures the value of the diffusion element is drawn for different time of exposure to chloride solution: 1, 10, 20 and 50 years. Comparing Figs. 3, 4, 5 and 6, it is clear that the value of the diffusion element of the circular model is smaller than the value of the diffusion element of the slab model for exposure time of 20 or more years. This difference increases with the decrease of the radius of the circular cross-section and the increase of the depth at which the ions diffusion is assessed.

Figure 7 shows the ratio of the chloride concentration inside the element and the chloride concentration on the exposed external surface as function of the depth at with it is estimated. From this figure it is clear that after around 10 years of exposure to the chloride solution the chloride concentration at a given depth in the concrete matrix from the external surface is higher for circular columns than for plane elements. This concentration difference that is clearly visible already at 10 years of exposure grows with the radius and the increase of depth at which the ratio is evaluated. The observation deduced from Fig. 7 is confirmed by the data collected in Tables 1, 2, 3 and 4.

As consequence of the results presented in the previous figure, Fig. 8 shows that the depassivation process begins earlier in the cylindrical columns than in the plane elements. Therefore, it is clear that for RC columns with small round cross-sections the life-time is shorter than the lifetime of plane RC members and this difference cannot be overlooked.

As said in the introduction section, the time to corrosion initiation $t_{c r}$ the instant at which the chloride concentration $C$ at the depth of the rebars in the concrete matrix exceeds the chloride threshold concentration for the depassivation $C_{c r}$. In this paper the threshold chloride concentration used for the simulations is $C_{c r}=0.2$, while the chloride concentration on the external surface is $C_{0}=0.4$. The final aim of this study is to give indications about the correct and judicious use of the standard slab model instead of the circular one for the chloride diffusion model in real RC elements. For this purpose the time to corrosion initiation of both circular columns with different radii and flat $\mathrm{RC}$ members is estimated and the values are collected in Table 5. Furthermore, the values of the critical time for the circular model are plotted on the left side of Fig. 9. The difference between the time to corrosion initiation estimated with the slab model and that time estimated with the circular model is shown on the right side of Fig. 9. In this figure the time to corrosion initiation is also estimated for three values of the diffusion coefficient of young concrete $D(0.63$, $0.32,0.16 \mathrm{~cm}^{3} /$ year $)$ corresponding to three different $w / c$ ratios $(0.50,0.45,0.40)$. From Fig. 9 and the data collected in Tables 5 and 6 it is clear the importance of the model used for the estimation of the chloride concentration for columns with radius smaller than $50 \mathrm{~cm}$ and a $w / c$ ratio lower than 0.5 in case of young OPC Type I. In fact, the model for the chloride diffusion in circular domain determines shorter critical time than the model for the diffusion in a semi-infinite plane. This difference cannot be overlooked both for the evaluation of the life time of single RC elements with circular cross-section and 
for the evaluation of the life time of whole RC structures with circular columns.

The chloride concentration on the external surface influences the estimation of the critical time because the velocity of the chloride ions diffusion increases with higher chloride concentration gradient. For low external concentrations the difference of the critical time estimated for circular columns with the different radii is lower, so in this case it is possible to use the diffusion model in a semi-infinite plane instead of the diffusion model in a circular domain. This result is shown in Fig. 10. By the observation of Fig. 10, an important conclusion can be drawn: the critical time is strongly influenced by the column radius for chloride concentration on the external surface higher than 0.25 . Therefore, in that case the model used to define the chloride diffusion has a significant influence.

Finally, all the data collected in all the tables and plotted in the figures suggest the following indication about the use of the slab model to estimate the time to corrosion initiation for cylindrical columns. In case of chloride concentration higher than 0.25 on the external surface of cylindrical RC column characterized by radius smaller than $50 \mathrm{~cm}$ the model to use necessarily to estimate the critical time to corrosion is the circular one. The error produced by the use of the slab model is large and should not be overlooked in the reliability analysis of existing RC columns. This practical and clear indication was missing in the literature.

\section{Conclusions}

The estimation of the time to corrosion initiation in aggressive environment is still a matter of study in life time assessment of RC structures. Many different mathematical models of the chloride diffusion in the concrete matrix were proposed in literature. These models are characterized by different complexity and number of parameters to be estimated, but most of them are related to the diffusion in a semi-infinite plane medium. This assumption is correct for $\mathrm{RC}$ plane members with only one surface exposed to a solution of chloride ions. It is inadequate for RC elements with different geometrical shapes, such as cylindrical elements. In RC elements with a circular cross-section the chloride penetration is faster than in plane elements, so the slab model used in literature overestimates the real time to corrosion initiation in case of cylindrical RC columns. In this paper an exact analytical model for the chloride penetration in RC elements with circular cross-section is used to evaluate the real time to corrosion initiation of these $\mathrm{RC}$ elements. In this study real values of the parameters characterizing the chloride diffusion in saturated condition and real dimensions of RC structural members are used to calculate the time to corrosion initiation of the rebars. The times to corrosion initiation calculated for plane and cylindrical RC members show the importance of the geometry for the correct assessment of the life time of a structure. In particular the results show that the circular model for the diffusion has to be used for columns with radius smaller the
$50 \mathrm{~cm}$ and for the external chloride concentration higher than 0.25 . This practical indication is directed to modify the standard approach conventionally used to estimate the critical time in RC circular columns. For this reason this study is an advance in the technical knowledge.

\section{Open Access}

This article is distributed under the terms of the Creative Commons Attribution License which permits any use, distribution, and reproduction in any medium, provided the original author(s) and the source are credited.

\section{Appendix}

Equation (5) can be solved with the method of the separation of variables:

$$
C(\rho, t)=\varphi(\rho) \psi(t)
$$

so the PDE (Eq. (12)) becomes

$$
\frac{\partial(\varphi(\rho) \psi(t))}{\partial t}=D\left(\frac{1}{\rho} \frac{\partial(\varphi(\rho) \psi(t))}{\partial \rho}+\frac{\partial^{2}(\varphi(\rho) \psi(t))}{\partial \rho^{2}}\right)
$$

The PDE (Eq. (13)) can be written as

$$
\frac{\varphi(\rho) \psi^{\prime}(t)}{D}=\psi(t)\left(\frac{1}{\rho} \varphi^{\prime}(\rho)+\varphi^{\prime \prime}(\rho)\right)
$$

Separating the functions $\psi(t)$ and $\varphi(\rho)$ and re-arranging the equation two differential equations equal to the constant $-\lambda^{2}$ are obtained:

$$
\frac{\psi^{\prime}(t)}{\psi(t)} \frac{1}{D}=\frac{1}{\rho} \frac{\varphi^{\prime}(\rho)}{\varphi(\rho)}+\frac{\varphi^{\prime \prime}(\rho)}{\varphi(\rho)}=-\lambda^{2}
$$

The equation can be written as a system of equations:

$$
\left\{\begin{array}{l}
\frac{\psi^{\prime}(t)}{\psi(t)} \frac{1}{D}=-\lambda^{2} \\
\frac{1}{\rho} \frac{\varphi^{\prime}(\rho)}{\varphi(\rho)}+\frac{\varphi^{\prime \prime}(\rho)}{\varphi(\rho)}=-\lambda^{2}
\end{array}\right.
$$

The first equation of Eq. (16) is a linear first order differential equation with the time as independent variable, while the second one is an ordinary second order differential equation with radial coordinate as independent variable and it is called Bessel's equation.

Inserting the boundary and initial conditions, Eqs. (6-8), the solution of Eq. (5) is (Saassouh and Lounis 2012)

$$
\begin{aligned}
C= & C_{0}\left(1-\frac{2}{R} \sum_{m=1}^{\infty} \frac{1}{\alpha_{m}} \frac{J_{0}\left(\rho \alpha_{m}\right)}{J_{1}\left(R \alpha_{m}\right)} e^{\left(-D \alpha_{m}^{2} t\right)}\right) \\
& +\frac{2}{R^{2}} \sum_{m=1}^{\infty} \frac{1}{\alpha_{m}} \frac{J_{0}\left(\rho \alpha_{m}\right)}{J_{1}^{2}\left(R \alpha_{m}\right)} e^{\left(-D \alpha_{m}^{2} t\right)} \int \rho f(\rho) J_{0}\left(\rho \alpha_{m}\right) d \rho
\end{aligned}
$$


where $\alpha_{m}$ are computed from the zeros of Bessel's first function with zero order

$$
J_{0}\left(\alpha_{m} R\right)=0
$$

and $J_{n}$ is the Bessel's first function with order $n$.

When the initial chloride concentration in the circular cross-section is constant $f(\rho)=C_{i n}$, Eq. (17) becomes

$$
C=\left(C_{0}-C_{i n}\right)\left(1-\frac{2}{R} \sum_{m=1}^{\infty} \frac{1}{\alpha_{m}} \frac{J_{0}\left(\rho \alpha_{m}\right)}{J_{1}\left(R \alpha_{m}\right)} e^{\left(-D \alpha_{m}^{2} t\right)}\right)+C_{i n}
$$

Finally, in case of concrete matrix with no chloride content at the time of exposure beginning $C_{i n}=0$ the solution of Eq. (19) of the diffusion equation is

$$
C=C_{0}\left(1-\frac{2}{R} \sum_{m=1}^{\infty} \frac{1}{\alpha_{m}} \frac{J_{0}\left(\rho \alpha_{m}\right)}{J_{1}\left(R \alpha_{m}\right)} e^{\left(-D \alpha_{m}^{2} t\right)}\right)
$$

\section{References}

Arora, P., Popov, B. N., Haran, B., Ramasubramanian, M., Popova, S., \& White, R. E. (1997). Corrosion initiation time of steel reinforcement in a chloride environment-A one dimensional solution. Corrosion Science, 39(4), 739-759.

Azad, A. K. (1998). Chloride diffusion in concrete and its impact on corrosion of reinforcement. In Proceedings of symposium on performance of concrete structures in the Arabian Gulf environment (pp. 262-273).

Basheer, L., Kropp, J., \& Cleland, D. J. (2002). Assessment of the durability of concrete from its permeation properties: A review. Construction and Building Materials, 15(2-3), 93-103.

Chatterji, S. (1995). On the applicability of the Fick's second law to chloride ion migration through Portland cement concrete. Cement and Concrete Research, 25(2), 299-303.

Collepardi, C. M., Marcialis, A., \& Turriziani, R. (1972). Penetration of chloride ions into cement pastes and concrete. Journal of the American Ceramic Society, 55(10), 534-535.

Crank, J. (1975). The mathematics of diffusion (2d ed.). London, UK: Oxford Press.

Do, J., Song, H., So, S., \& Soh, Y. (2005). Comparison of deterministic calculation and fuzzy arithmetic for two prediction model equation of corrosion initiation. Journal of Asian Architecture and Building Engineering., 4(2), 447-454.
Frier, C, \& Sørensen, J. D. (2007). Stochastic analysis of the multi-dimensional effect of chloride ingress into reinforced concrete. In 10th international conference of applications of statistics and probability in civil engineering (pp. 135-136), Tokyo, Japan, 31 July-3 August 2007. London, UK: Marcel Dekker.

Izquierdo, D., Alonso, C., Andrade, C., \& Castellote, M. (2004). Potenziostatic determination of chloride threshold values for rebar depassivation. Experimental and Statistical Study, Electrochimica Acta, 49, 2731-2739.

Kirkpatrick, T. J., Weyers, R. E., Sprinkel, M. M., \& AndersonCook, C. M. (2002). Impact of specification changes on chloride-induced corrosion service life of bridge decks. Cement and Concrete Research, 32(8), 1189-1197.

Lin, S. H. (1990). Chloride diffusion in a porous concrete slab. Corrosion, 46, 964-967.

Marano, G. C., Quaranta, G., \& Mezzina, M. (2008). Fuzzy Time-dependent reliability analysis of RC beams subject to pitting corrosion. Journal of Materials in Civil Engineering, 20(9), 578-587.

Martin-Perez, B., Pantazopoulou, S. J., \& Thomas, M. D. A. (2001). Numerical solution of mass transport equations in concrete structures. Computers \& Structures, 79(13), 1251-1264.

Nogueira, C. G., \& Leonel, E. D. (2013). Probabilistic models applied to safety assessment of reinforced concrete structures subjected to chloride ingress. Engineering Failure Analysis, 31, 76-89.

Saassouh, B., \& Lounis, Z. (2012). Probabilistic modelling of chloride-induced corrosion in concrete structures using first- and second-order reliability methods. Cement \& Concrete Composites, 34, 1082-1093.

Saetta, A. V., Schrefler, B. A., \& Vitaliani, R. V. (1995). 2-D model for carbonation and moisture/heat flow in porous materials. Cement and Concrete Research, 25(8), 1703-1712.

Sobhani, J., \& Ramezanianpour, A. A. (2011). Service life of the reinforced concrete bridge deck in corrosive environments: A soft computing system. Applied Soft Computing, 11, 3333-3346.

Tang, L., \& Joost, G. (2007). On the mathematics of timedependent apparent chloride diffusion coefficient in concrete. Cement and Concrete Research, 37, 589-595.

Tuutti, K. (1982). Corrosion of steel in concrete. Swedish Cement and Concrete Research Institute, Stockholm, Sweden. Report No. CBI Research FO 4:82.

Vu, K. A. T., \& Stewart, M. G. (2000). Structural reliability of concrete bridges including improved chloride-induced corrosion models. Structural Safety, 22(4), 313-333.

Val, D. V., \& Trapper, P. A. (2008). Probabilistic evaluation of initiation time of chloride-induced corrosion. Reliability Engineering and System Safety, 93(3), 364-372. 